\title{
Weniger Parodontitis durch mehr und bessere Behandlung!?
}

Die Ergebnisse der 5. Deutschen Mundgesundheitsstudie (DMS V) wurden gerade veröffentlicht. Was hat sich an der parodontalen Gesundheit der Deutschen in 10 Jahren verändert? Bei den jungen Erwachsenen (35-44 Jahre) ist der Anteil von CPICode 3 (Taschentiefen von mindestens 4 $\mathrm{mm}$ ) von 52,7\% in der DMS IV von 2005 auf 48,3\% in 2015 zurückgegangen. Deutlicher ist der Rückgang bei den schweren Parodontalerkrankungen (CPI 4: Taschentiefen $\geq 6 \mathrm{~mm}$ ) von $20,5 \%$ auf $10,4 \%$ in 2015. Nach Korrektur für die Unterschätzung der Erhebung an nur einem Teil der Zähne sind es dann allerdings doch wie- der 14,5\% (Hoffmann \& Schützhold 2016)! Bei den Senioren (65-74 Jahre) ist das Bild nicht ganz so eindeutig. Hier nehmen die moderaten Parodontalerkrankungen (CPI 3) von $48 \%$ (DMS IV) auf 50,8 \% etwas zu. Die schweren Parodontalerkrankungen der über 65-Jährigen sind aber ebenfalls rückläufig, von 39,8 (DMS IV) auf 24,6\% (Kocher \& Holtfreter 2016). Das ist insgesamt eine großartige Entwicklung.

Nach einer Pressemitteilung der Deutsche Gesellschaft für Parodontologie e.V., Regensburg www.dgparo.de 